
filament embedded in an isotropic elastic matrix

Stephen G. O'Keeffe

Derek E. Moulton

Sarah L. Waters

Alain Goriely 



\title{
Growth-induced axial buckling of a slender elastic filament embedded in an isotropic elastic matrix
}

\author{
Stephen G. O'Keeffe, Derek E. Moulton, Sarah L. Waters and Alain Goriely \\ Mathematical Institute, University of Oxford
}

April 21, 2013

\begin{abstract}
We investigate the problem of an axially-loaded, isotropic, slender cylinder embedded in a soft, isotropic, outer elastic matrix. The cylinder undergoes uniform axial growth, whilst both the cylinder and surrounding elastic matrix are confined between two rigid plates, so that this growth results in axial compression of the cylinder. We use two different modelling approaches to estimate the critical axial growth (that is, the amount of axial growth the cylinder is able to sustain before it buckles) and buckling wavelength of the cylinder. The first approach treats the filament and surrounding matrix as a single 3-dimensional elastic body undergoing large deformations, whilst the second approach treats the filament as a planar, elastic rod embedded in an infinite elastic foundation. By comparing the results of these two approaches, we obtain an estimate of the foundation modulus parameter, which characterises the strength of the foundation, in terms of the geometric and material properties of the system.
\end{abstract}

\section{Introduction}

Cylindrical structures are ubiquitous throughout the biological world. Examples can be found across a broad range of length scales, from microtubules within the cell to macroscopic plant stems. Every type of structure fulfils a key role within its relevant environment, such as oxygen and nutrient transport provided by airways and arteries, or signal transmission carried out by axons. Biological structures are often subject to differential growth, a process whereby different regions of tissue within the same body grow at different rates. This gives rise to residual stresses that exist in the absence of any applied tractions, and have important effects on the overall mechanical behaviour of a material body [7,8]. Should the stresses within a body become sufficiently large, material failure can occur and this may be manifested in various ways, including fracture, cracking, plastic yield or buckling.

Rod and beam-like structures are often embedded within another material. Examples include plant roots growing in soil, microtubules embedded within the cytoplasm, and blood vessels surrounded by body tissue. A simple modelling approach that can be applied to each of these scenarios is to treat the surrounding material as an elastic foundation. Many studies of such embedded slender structures make use of the classical Euler beam theory [4] in order to study the onset of buckling under the action of a compressive force. Murmu and Pradhan [17] used Timoshenko beam theory (an extension of Euler beam theory) to investigate the effect of various types of elastic foundation on the critical longitudinal buckling stress (i.e. the compressive stress at which the tube buckles) of embedded single-walled carbon nanotubes. Their analysis made use of a foundation modulus parameter, $k_{f}$, which characterises the amount of tranverse reinforcement of the foundation acting on the tube $[24,23,5]$. They found that the critical buckling pressure increases with $k_{f}$, although no information is given on how the parameter $k_{f}$ can be measured for a specific material. Brangwynne et al. performed buckling experiments on microtubules. They applied axial loads to microtubules under two different circumstances. Firstly, when excised from the cell they observed that under small axial loads, microtubules exhibited Euler-type buckling [3]. However, when embedded within the cytoplasm they were able to withstand a greater compressive force 
(imposed by applying a normal force at the point where the microtubule meets the cell membrane) before the onset of buckling. Moreover, once buckling did occur, the observed wavelength was shorter than when isolated microtubules were considered. The authors also carried out a theoretical analysis by treating a microtubule as a cylindrical, inextensible beam embedded within an elastic foundation. They proposed that the observed buckling mode is that which minimises the sum of the beam's bending energy and the energy required to transversely displace the surrounding cytoplasm. Their analysis uses the parameter $\alpha$ to characterise the transverse reinforcement of the cytoplasm, and they estimate this parameter in terms of the radius of the rod, $a$, the Young's modulus of the surrounding matrix, $G$, and the characteristic buckling length scale of the structure, $l$ as follows:

$$
\alpha_{e 1}=\frac{4 \pi G}{\log \left(\frac{l}{a}\right)} .
$$

However, this approach is problematic, since the wavelength $l$ is not known a a priori. Therefore this estimate cannot be used in any predictive way. Furthermore, its derivation remains mysterious. An alternative estimate of this parameter where the wavelength is replaced by the length $L$ of the beam was presented in [20], and given by

$$
\alpha_{e 2}=\frac{4 \pi G}{\log \left(\frac{L}{a}\right)} .
$$

As we will see, this estimate cannot be correct either as the buckling properties of a long filament in a matrix are essentially independent of the length of the beam. By comparing the exact buckling properties of a cylinder under axial load in an infinite matrix with the properties of a Kirchhoff rod on an elastic foundation we derive a new estimate for the foundation modulus parameter.

\subsection{The Problem}

We consider the problem of an isotropic, slender, elastic filament embedded in an isotropic, elastic matrix. The filament and matrix are constrained in the axial direction by two flat, rigid plates and the filament undergoes uniform axial growth, so that it becomes axially compressed. At a critical growth, the filament buckles. We model this problem firstly by considering volumetric growth in a 3-dimensional, non-linear elastic body. This approach has been used in a number of previous studies of axial and circumferential buckling of hollow multi-layered cylinders with finite radii $[10,21,22,14]$, and exploits the idea of multiplicative decomposition of the deformation tensor into two components: stress-free growth and elastic response. The framework was originally proposed by Rodriguez et al. [19], and has since been widely incorporated into many models of volumetric growth, e.g. [14]. For the case of a neo-Hookean filament and neo-Hookean matrix we are able to estimate the critical growth value analytically via the use of the WKB method. Secondly, we consider a rod-theory formulation that models the system as a two-dimensional elastic beam embedded in a Winkler foundation. Like previous rod-theory models, this approach makes use of a foundation modulus parameter. Each approach provides us with an estimate of the critical axial growth of the filament and the wavelength of the resulting buckled state. The goal of this paper is to fully describe this buckling instability and obtain an estimate of this parameter by comparing the results of two approaches. This foundation modulus parameter is directed related to geometric and material properties of the system. Further, the generalisation to a Mooney-Rivlin matrix does not alter this estimate.

\section{3-D Elasticity Approach}

\subsection{General Setup}

We follow closely the method outlined in [1]. Consider an elastic body occupying a reference (stressfree) configuration, $\mathcal{B}_{0}$, defined by co-ordinates $\mathbf{X}$. The body is subject to a deformation, $\chi$, so that its new configuration, $\mathcal{B}_{f}$ (which we shall refer to as the current configuration), is defined by co-ordinates $\mathbf{x}=\boldsymbol{\chi}(\mathbf{X})$. Let $\mathbf{F}=d \boldsymbol{\chi} / d \mathbf{X}=\operatorname{Grad}(\boldsymbol{\chi})$ be the geometric deformation tensor.

As described in the Introduction, the effects of growth are incorporated into the model via multiplicative decomposition of the deformation gradient tensor, so that $\mathbf{F}=\mathbf{A} \mathbf{G}$, where $\mathbf{G}$ represents local 
stress-free growth and A represents the elastic response. Since we will restrict our attention to semiinverse problems where cylinders are mapped onto other cylinders, a full description of the kinematics of growth is not required.

We assume that the body is incompressible, which implies that only isochoric deformatiosn are possible, that is

$$
\operatorname{det} \mathbf{A}=1
$$

We also assume the body is hyperelastic, that is there exists a strain-energy density, $W$, such that

$$
\mathbf{T}=\mathbf{A} \frac{\partial W}{\partial \mathbf{A}}-p \mathbb{1}
$$

where $\mathbf{T}$ is the Cauchy stress tensor and $p$ is a Lagrange multiplier enforcing the incompressibility constraint. In the absence of body forces, the equation of static mechanical equilibrium can be written as

$$
\operatorname{div} \mathbf{T}=\mathbf{0}
$$

For our problem, a natural choice for boundary conditions is to prescribe the deformation on some part of the boundary and impose a pressure, $P$, acting in the normal direction, $\hat{\mathbf{n}}$, on the rest of the boundary:

$$
\mathbf{T} . \hat{\mathbf{n}}=-P \hat{\mathbf{n}} .
$$

\subsection{Incremental Deformations}

We now investigate the stability of solutions of (5). We do so by introducing a small perturbation to the finite deformation. This perturbation is an incremental deformation, that belongs to a wider class of deformation with no prescribed symmetry, and is defined by

$$
\chi(\mathbf{X})=\chi^{(0)}(\mathbf{X})+\epsilon \chi^{(1)}(\mathbf{X})
$$

where $0<\epsilon \ll 1$ characterises the size of the perturbation. Accordingly, we define

$$
\mathbf{F}=\left(\mathbb{1}+\epsilon \mathbf{F}^{(1)}\right) \mathbf{F}^{(0)}, \quad \mathbf{A}=\left(\mathbb{1}+\epsilon \mathbf{A}^{(1)}\right) \mathbf{A}^{(0)} .
$$

The incremental equations and boundary conditions are formulated in terms of the current configuration. We expand the Cauchy stress tensor as follows

$$
\mathbf{T}=\mathbf{T}^{(0)}+\epsilon \mathbf{T}^{(1)}+O\left(\epsilon^{2}\right)
$$

Substitution of (9) into (4), gives

$$
\begin{aligned}
& \mathbf{T}^{(0)}=\mathbf{A}^{(0)} \frac{\partial W^{(0)}}{\partial \mathbf{A}^{(0)}}, \\
& \mathbf{T}^{(1)}=\mathcal{L}: \mathbf{A}^{(1)}+\mathbf{A}^{(1)} \mathbf{A}^{(0)} W_{A}^{(0)}-p^{(1)} \mathbb{1},
\end{aligned}
$$

where $p=p^{(0)}+\epsilon p^{(1)}$ and $\mathcal{L}$ is the fourth-order tensor given by

$$
\mathcal{L}: \mathbf{A}^{(1)}=\mathbf{A}^{(0)}\left(W_{A A}^{(0)}: \mathbf{A}^{(1)}\right) \mathbf{A}^{(0)}
$$

Here, $W_{A}^{(0)}$ and $W_{A A}^{(0)}$ are the first and second derivatives of $W$ with respect to $\mathbf{A}$ evaluated at $\mathbf{A}^{(0)}$. Explicitly, the non-zero components of $\mathcal{L}$ are given by [18]:

$$
\left.\begin{array}{rll}
\mathcal{L}_{i i j j} & =\mathcal{L}_{j j i i}=\alpha_{i} \alpha_{j} \frac{\partial^{2} W}{\partial \alpha_{i} \partial \alpha_{j}}, & \\
\mathcal{L}_{i j i j} & =\alpha_{i}^{2} \frac{\alpha_{i} \frac{\partial W}{\partial \alpha_{i}}-\alpha_{j} \frac{\partial W}{\partial \alpha_{j}}}{\alpha_{i}^{2}-\alpha_{j}^{2}}, & \\
\mathcal{L}_{i j i j} & =\frac{\mathcal{L}_{i i i i}-\mathcal{L}_{i i j j}+\alpha_{i} \frac{\partial W}{\partial \alpha_{i}}}{2}, & i \neq j, \alpha_{i} \neq \alpha_{j}, \\
-\mathcal{L}_{i j j i} & =\mathcal{L}_{i j i j}-\stackrel{\mathcal{L}}{j i i j}_{i j}=\alpha_{i} \frac{\partial W}{\partial \alpha_{i}}, & i \neq j,
\end{array}\right\}
$$

where $\alpha_{i}$ are the principal values of $\mathbf{A}^{(0)}$. The equilibirum equations are then given by

$$
\begin{aligned}
& \operatorname{div}\left(\mathbf{T}^{(0)}\right)=\mathbf{0}, \\
& \operatorname{div}\left(\mathbf{T}^{(1)}\right)=\mathbf{0} .
\end{aligned}
$$




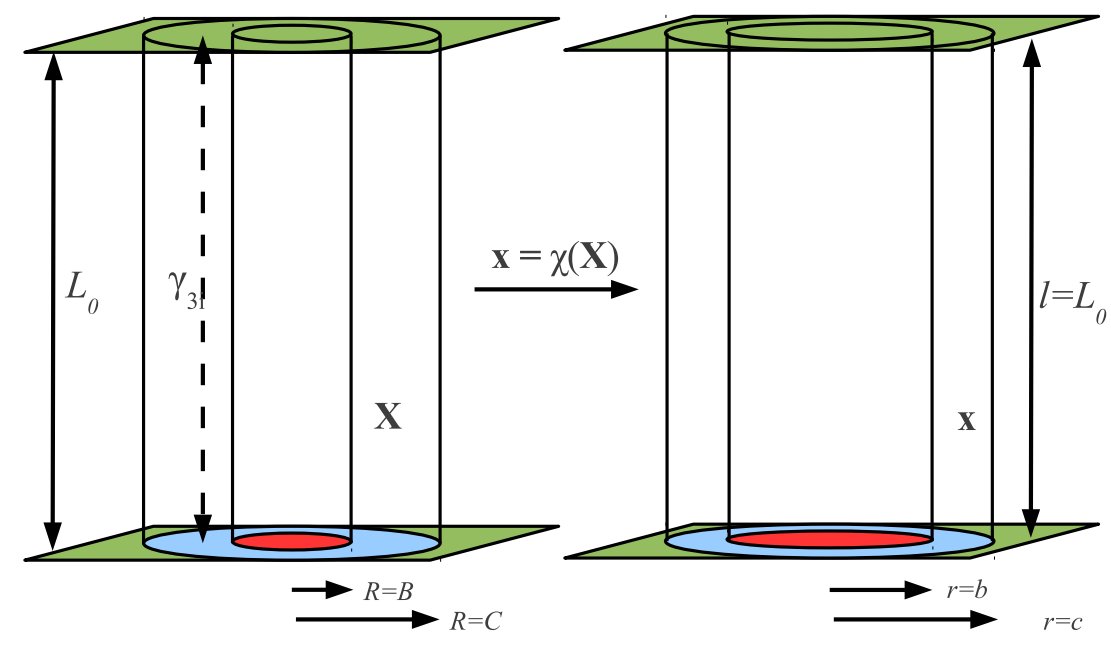

Figure 1: Illustration of the axisymmetric deformation of a two-layer cylinder subject to axial differential growth. Capitals denote quantities defined with the respect to the reference configuration, $\mathcal{B}_{0}$ (left), while lower case characters denote the same quantities defined in terms of the current configuration, $\mathcal{B}_{f}$ (right). We are primarily interested in the zero-extension case, therefore we set $l=L_{0}$.

The boundary conditions are given by fixing $\chi^{(0)}$ and $\chi^{(1)}$ on some part of the boundary and on the remainder, substitution into (6) gives

$$
\begin{aligned}
& \mathbf{T}^{(0)} \cdot \hat{\mathbf{n}}=\mathbf{0}, \\
& \mathbf{T}^{(1)} \cdot \hat{\mathbf{n}}=\mathbf{0} .
\end{aligned}
$$

The existence of a solution to (15) and prescribed boundary conditions indicates a bifurcation and suggest that an instability is possible. We shall assume throughout this paper that the existence of such a solution renders the solution $\chi^{(0)}$ unstable.

\subsection{The Two-Layer Cylinder}

Having set up the general finite and incremental equilibrium equations and boundary conditions, we use them to solve our specific problem. We consider a two-layer, solid cylinder constrained axially between two rigid plates. The reference configuration, $\mathcal{B}_{0}$, is defined by co-ordinates $\mathbf{X}=R \mathbf{e}_{R}+Z \mathbf{e}_{Z}$, with $0 \leq R \leq C, 0 \leq Z \leq L_{0}$ and a material boundary at $R=B$. Following the deformation, the cylinder occupies the configuration $\mathcal{B}_{f}$ defined by co-ordinates $\mathbf{x}=\chi^{(0)}(\mathbf{X})=r(R) \mathbf{e}_{R}+\lambda_{z} Z \mathbf{e}_{Z}$, with $0 \leq r \leq c$ and $0 \leq z \leq l$. The parameter $\lambda_{z}=l / L_{0}$ is a constant, representing the overall change in length of the cylinder and we use $b$ and $c$ to denote the material boundaries in the deformed configuration, i.e. $b=r(B)$ and $c=r(C)$. The presence of the rigid plates requires that $l=L_{0}$ so that $\lambda_{z}=1$. This deformation is shown schematically in Figure 1. We prescribe constant axial growth in the inner layer, denoted by the parameter $\gamma$ and allow no growth or resorption to occur in the other two directions, nor in any direction in the outer layer. 


\subsubsection{Axisymmetric Deformations}

We first consider the mechanics and geometry of the axisymmetric solution prior to bifurcation. The deformation gradient tensor is then given by

$$
\mathbf{F}^{(0)}=\operatorname{diag}\left(r^{\prime}, \frac{r}{R}, 1\right)
$$

where the prime denotes differentiation with respect to $R$. The growth tensor is given by

$$
\mathbf{G}=\operatorname{diag}\left(1,1, \gamma_{3}\right), \quad \gamma_{3}= \begin{cases}\gamma, & 0 \leq R \leq B \\ 1, & B<R \leq C,\end{cases}
$$

and the elastic response tensor is given by

$$
\mathbf{A}^{(0)}=\operatorname{diag}\left(\alpha_{1}, \alpha_{2}, \alpha_{3}\right) \text {. }
$$

From (19)-(20) and the relationship $\mathbf{F}^{(0)}=\mathbf{A}^{(0)} \mathbf{G}$, the variables $\alpha_{j}$ are given by $\alpha_{1}=r^{\prime}, \quad \alpha_{2}=$ $r / R, \quad \alpha_{3}=1 / \gamma_{3}$. Initially, we shall assume both layers are neo-Hookean, and we define the strainenergy density in each layer to be

$$
W_{i, o}\left(\alpha_{1}, \alpha_{2}, \alpha_{3}\right)=\frac{\mu_{i, o}}{2}\left(\alpha_{1}^{2}+\alpha_{2}^{2}+\alpha_{3}^{2}-3\right),
$$

where $\mu_{i, o}$ are the shear moduli of each layer. Utilising the incompressibility condition (3), it follows that $\alpha_{1} \alpha_{2} \alpha_{3}=1$, thus we may define $\hat{W}_{i, o}$ such that

$$
W_{i, o}\left(\frac{1}{\alpha_{2} \alpha_{3}}, \alpha_{2}, \alpha_{3}\right)=\hat{W}_{i, o}\left(\alpha_{2}, \alpha_{3}\right)=\frac{\mu_{i, o}}{2}\left(\frac{1}{\alpha_{2}^{2} \alpha_{3}^{2}}+\alpha_{2}^{2}+\alpha_{3}^{2}-3\right) .
$$

Since $\mathbf{A}^{(0)}$ is diagonal, it follows that $\mathbf{T}^{(0)}=\operatorname{diag}\left(t_{r r}, t_{\theta \theta}, t_{z z}\right)$ and substitution into (5) yields only one non-trivial equation:

$$
\frac{d t_{r r}}{d r}+\frac{t_{r r}-t_{\theta \theta}}{r}=0
$$

At the boundary between the inner and outer layer $(r=b)$, we require the displacement to be continuous. Furthermore, the radial stress must be continuous. On the outer radius of the cylinder $(r=c)$, we impose the condition (16) with $P=0$ and $\hat{\mathbf{n}}=\mathbf{e}_{r}$, whilst at the centre we require the radial stress to remain bounded. Therefore, the boundary conditions are as follows

$$
\begin{aligned}
t_{r r}(c) & =0, \\
t_{r r}(0) & <\infty, \\
{\left[t_{r r}\right]_{r=b} } & =0 .
\end{aligned}
$$

Note that it may not be possible to satisfy (25). In such cases the deformed body will contain a cavity and (25) is replaced by a zero radial stress condition on the inner wall, i.e. $t_{r r}(a)=0$, where $a=r(0)$, as detailed in [9]. Here, we do not consider the possibility of cavitation.

Substitution of (22) into (10) and (23) then gives

$$
\frac{d t_{r r}}{d r}=\frac{\alpha_{2}}{r} \frac{\partial \hat{W}_{i, o}}{\partial \alpha_{2}}
$$

which can be integrated to yield

$$
t_{r r}(r)= \begin{cases}t_{r r}(b)-\int_{b}^{r} \frac{\alpha_{2}}{r} \frac{\partial \hat{W}_{i}}{\partial \alpha_{2}} \mathrm{~d} r, & 0 \leq r \leq b, \\ -\int_{c}^{r} \frac{\alpha_{2}}{r} \frac{\partial \hat{W}_{o}}{\partial \alpha_{2}} \mathrm{~d} r, & b \leq r \leq c .\end{cases}
$$

The incompressibility equation (3) can be integrated directly to give

$$
r= \begin{cases}\sqrt{\gamma} R, & 0 \leq R \leq B \\ \sqrt{b^{2}+\left(R^{2}-B^{2}\right)}, & B \leq R \leq C .\end{cases}
$$




\subsubsection{Incremental Equations}

We denote the components of the incremental deformation, $\chi^{(1)}$, in polar co-ordinates as $(u(r, \theta, z), v(r, \theta, z), w(r, \theta, z))^{T}$. This gives the following expression for $\mathbf{A}^{(1)}$ :

$$
\mathbf{A}^{(1)}=\operatorname{grad} \boldsymbol{\chi}^{(1)}=\left(\begin{array}{ccc}
u_{r} & \frac{u_{\theta}-v}{r} & u_{z} \\
v_{r} & \frac{u+v_{\theta}}{r} & v_{z} \\
w_{r} & \frac{w_{\theta}}{r} & w_{z}
\end{array}\right),
$$

where subscripts denote differentiation with respect to the relevant variable. The incompressibility condition leads to the relation

$$
\operatorname{tr} \mathbf{A}^{(1)}=u_{r}+\frac{u+v_{\theta}}{r}+w_{z}=0
$$

We seek solutions of (15) that are Fourier modes in $\theta$ and $z$ :

$$
\begin{aligned}
u & =f(r) \cos (m \theta) \cos (\alpha z), \\
v & =g(r) \sin (m \theta) \cos (\alpha z), \\
w & =h(r) \cos (m \theta) \sin (\alpha z), \\
p_{1} & =k(r) \cos (m \theta) \cos (\alpha z),
\end{aligned}
$$

where $\alpha=n \pi / L_{0}, n \in \mathbb{N}$ and $m$ is the mode number, chosen to be a non-negative integer so that displacements are single-valued. The values of $m$ and $n$ determine the type of buckling that occurs. If $m=0$ and $n \geq 1$, the centreline of the cylinder remains straight and we obtain radially symmetric solutions, but a 'barrelling' effect is observed. If $m=1$ and $n \geq 1$ then the cross-section of the cylinder remains circular, however the centreline ceases to be straight. Finally, if $m \geq 2$ we observe circumferential buckling, with $m$ nodes visible in the cross-section. Axial oscillations are possible when $n \geq 1$. Each of these cases has been studied previously. For example, Moulton and Goriely examined the case of cross-sectional buckling $(m \geq 1, n=0)$ as a result of radial growth [15]. MacLaurin et al. studied combinations of axial and circumferential modes $m \geq 1, n \geq 1$ arising in tumour capillaries due to the rapid growth of malignant cells [13]. Goriely and Vandiver investigated the axial stability of growing arteries $(m=1, n \geq 1)$ [10]. In 2008, Goriely et al studied the effect of geometry on the type of buckling observed for a one-layer, neo-Hookean cylinder [11]. They found that in the regime of a slender, solid cylinder, instability will always develop in the axial direction before the circumferential direction. Using this information, we make the ansatz that, in the two-layer case, an axial buckling mode will be the observed buckling mode, therefore we set $m=1$.

Substitution into the incremental equilibrium equations then yields

$$
\begin{aligned}
k^{\prime} & =\left(r\left(\mathcal{L}_{2211}^{\prime}-\mathcal{L}_{3311}^{\prime}\right)-\mathcal{L}_{2222}+\mathcal{L}_{2233}-\mathcal{L}_{2121}+\mathcal{L}_{1331}\right) g / r^{2} \\
& +\left(\mathcal{L}_{2112}+\mathcal{L}_{2211}-\mathcal{L}_{1331}-\mathcal{L}_{3311}\right) g^{\prime} / r+\left(\mathcal{L}_{1111}-\mathcal{L}_{3311}-\mathcal{L}_{1331}\right) f^{\prime \prime} \\
& +\left(r\left(\mathcal{L}_{1111}^{\prime}+p_{0}^{\prime}-\mathcal{L}_{3311}^{\prime}\right)+\mathcal{L}_{1111}-2 \mathcal{L}_{3311}+\mathcal{L}_{2233}-\mathcal{L}_{1331}\right) f^{\prime} / r \\
& +\left(r\left(\mathcal{L}_{2211}^{\prime}-\mathcal{L}_{3311}^{\prime}\right)-\mathcal{L}_{2222}+\mathcal{L}_{2233}+\mathcal{L}_{1331}\right) f / r^{2}-\mathcal{L}_{2121} f / r^{2}-\alpha^{2} \mathcal{L}_{3131} f, \\
r \mathcal{L}_{1212} g^{\prime \prime} & =-k+\left(r \mathcal{L}_{1212}^{\prime}+\mathcal{L}_{1212}+\mathcal{L}_{2222}-\mathcal{L}_{2233}-\mathcal{L}_{3223}\right) f / r \\
& +\left(r \mathcal{L}_{1212}^{\prime}+\mathcal{L}_{1212}\right) g / r+\left(\mathcal{L}_{2222}-\mathcal{L}_{2233}-\mathcal{L}_{3223}\right) g / r \\
& +r \alpha^{2} \mathcal{L}_{3232} g+\left(\mathcal{L}_{2211}-\mathcal{L}_{2233}-\mathcal{L}_{3223}+\mathcal{L}_{1221}\right) f^{\prime}-\left(r \mathcal{L}_{1212}+\mathcal{L}_{1212}\right) g^{\prime} \\
\mathcal{L}_{1313} f^{\prime \prime \prime} & =\left(f^{\prime}+(g+f) / r\right) \mathcal{L}_{2323} / r^{2}-\left(r \mathcal{L}_{1331}^{\prime}+r p_{0}^{\prime}+\mathcal{L}_{1331}-\mathcal{L}_{2332}\right) \alpha^{2} f / r \\
& -\mathcal{L}_{1313}\left(\left(f^{\prime \prime}+g^{\prime \prime}\right) / r-2\left(f^{\prime}+g^{\prime}\right) / r^{2}+2(f+g) / r^{3}\right) \\
& +\left(\mathcal{L}_{3333}-\mathcal{L}_{2332}-\mathcal{L}_{2233}\right) \alpha^{2}(f+g) / r+\left(\mathcal{L}_{3333}-\mathcal{L}_{3311}-\mathcal{L}_{1331}\right) \alpha^{2} f^{\prime} \\
& -\left(r \mathcal{L}_{1313}^{\prime}+\mathcal{L}_{1313}\right)\left(f^{\prime \prime}+\left(f^{\prime}+g^{\prime}\right) / r-(f+g) / r^{2}\right) / r-\alpha^{2} k .
\end{aligned}
$$


The incremental ambient pressure is zero, so applying (17) we have the following three boundary conditions at $r=c$

$$
\begin{aligned}
& \left(\mathcal{L}_{1122}-\mathcal{L}_{1133}\right)(f+g)+\left(\mathcal{L}_{1111}-\mathcal{L}_{1133}+\alpha_{1} \frac{\partial W}{\partial \alpha_{1}}\right) r f^{\prime}-r k=0, \\
& r g^{\prime}-g-f=0 \\
& r^{2} f^{\prime \prime}+r f^{\prime}+\alpha^{2} r^{2} f=0 .
\end{aligned}
$$

At the interface between the two layers $(r=b)$, we assume a continuous deformation without slippage, thus we require

$$
\begin{aligned}
f_{i} & =f_{o}, \\
g_{i} & =g_{o}, \\
f_{i}^{\prime} & =f_{o}^{\prime} .
\end{aligned}
$$

Furthermore, we require the radial stress at the interface to be continuous. Therefore

$$
\begin{aligned}
& \left(\mathcal{L}_{1122_{i}}-\mathcal{L}_{1133_{i}}\right)\left(f_{i}+g_{i}\right)+\left(\mathcal{L}_{1111_{i}}-\mathcal{L}_{1133_{i}}+\alpha_{1_{i}} \frac{\partial W_{i}}{\partial \alpha_{1_{i}}}\right) r f_{i}^{\prime}-r k_{i} \\
& =\left(\mathcal{L}_{112_{o}}-\mathcal{L}_{1133_{o}}\right)\left(f_{o}+g_{o}\right)+\left(\mathcal{L}_{1111_{o}}-\mathcal{L}_{1133_{o}}+\alpha_{1_{o}} \frac{\partial W_{o}}{\partial \alpha_{1_{o}}}\right) r f_{o}^{\prime}-r k_{o}, \\
& \mathcal{L}_{1212_{i}}\left(r g_{i}^{\prime}-g_{i}-f_{i}\right)=\mathcal{L}_{1212_{o}}\left(r g_{o}^{\prime}-g_{o}-f_{o}\right), \\
& \mathcal{L}_{1313_{i}}\left(r^{2} f_{i}^{\prime \prime}+r f_{i}^{\prime}+\left(\alpha^{2} r^{2}-1\right) f_{i}+r g_{i}^{\prime}-g_{i}\right) \\
& =\mathcal{L}_{1313_{o}}\left(r^{2} f_{o}^{\prime \prime}+r f_{o}^{\prime}+\left(\alpha^{2} r^{2}-1\right) f_{o}+r g_{o}^{\prime}-g_{o}\right) .
\end{aligned}
$$

Finally, we demand that all solutions remain bounded as $r \rightarrow 0$.

\subsection{Numerical Solutions}

We simplify equations (36)-(38) by eliminating $k$. This results in a six-dimensional system of equations that can be written in the form

$$
\mathbf{y}^{\prime}=\mathbf{f}(\mathbf{y} ; r), \quad \mathbf{y} \in \mathbb{R}^{6}
$$

where $\mathbf{y}=\left(f, f^{\prime}, f^{\prime \prime}, g, g^{\prime}, g^{\prime \prime}\right)$. The radial stresses must remain bounded as $r \rightarrow 0$. Therefore the functions $f, g$ and $k$ must be 'well-behaved' as $r \rightarrow 0$, i.e. we demand bounded approximations of $f, g$ and $k$ close to zero for which the incremental equilibrium equations are satisfied. We proceed by eliminating $k$ from (36)-(38) and seek $f$ and $g$ of the form

$$
f=Q_{1} r^{\nu_{1}}, \quad g=Q_{2} r^{\nu_{2}} .
$$

We substitue these expressions into (36)-(38) and eliminate the terms that do not decay as $r \rightarrow 0$ by setting the coefficients of such terms to be zero. There are three cases to consider; they are $\nu_{1}>\nu_{2}$, $\nu_{1}=\nu_{2}$ and $\nu_{1}<\nu_{2}$. Each gives rise to an admissible expression for both $f$ and $g$. Combining these expressions then yields

$$
f \sim a_{1} r^{2}+b_{1}, \quad g \sim a_{2} r^{2}-b_{1}, \quad \text { as } r \rightarrow 0,
$$

where $a_{1}, a_{2}$ and $b_{1}$ are constants. The remaining boundary conditions comprise a set of 9 linear functions $c_{1, \ldots, 9}(\mathbf{y}(r) ; r)$ :

$$
c_{1,2,3}\left(\mathbf{y}_{o}(c) ; c\right)=0, \quad c_{4,5,6,7,8,9}\left(\mathbf{y}_{i}(b) ; b\right)=c_{4,5,6,7,8,9}\left(\mathbf{y}_{o}(b) ; b\right) .
$$

The determinant method can be used to find values of $\gamma$ for which non-trivial solutions exist. We make three copies of the system (48) with linearly independent conditions of the form (50) as $r \rightarrow 0$. For our system, $\mathbf{y}^{(i)}, \quad i=1,2,3$, are given by

$$
\mathbf{y}^{(i)} \sim\left(f^{(i)}, f^{(i) \prime}, f^{(i) \prime \prime}, g^{(i)}, g^{(i) \prime}, g^{(i) \prime \prime}\right), \quad r \rightarrow 0,
$$


where

$$
\begin{gathered}
f^{(1)}=r^{2}, \quad g^{(1)}=0, \\
f^{(2)}=1, \quad g^{(2)}=-1, \\
f^{(3)}=0, \quad g^{(3)}=r^{2} .
\end{gathered}
$$

We integrate this system up to $r=b$ and use the boundary conditions at the interface (51(b)), together with the end values $\mathbf{y}_{i}^{(j)}(b), \quad j=1,2,3$, to obtain the initial conditions $\mathbf{y}_{o}^{(j)}(b), \quad j=1,2,3$. We then integrate up to $r=c$ and evaluate the $3 \times 3$ determinant of boundary conditions at $r=c$ given by

$$
\Delta(\gamma)=\left|\begin{array}{lll}
c_{1}\left(\mathbf{y}_{o}^{(1)}(c) ; \gamma\right) & c_{2}\left(\mathbf{y}_{o}^{(1)}(c) ; \gamma\right) & c_{3}\left(\mathbf{y}_{o}^{(1)}(c) ; \gamma\right) \\
c_{1}\left(\mathbf{y}_{o}^{(2)}(c) ; \gamma\right) & c_{2}\left(\mathbf{y}_{o}^{(2)}(c) ; \gamma\right) & c_{3}\left(\mathbf{y}_{o}^{(2)}(c) ; \gamma\right) \\
c_{1}\left(\mathbf{y}_{o}^{(3)}(c) ; \gamma\right) & c_{2}\left(\mathbf{y}_{o}^{(3)}(c) ; \gamma\right) & c_{3}\left(\mathbf{y}_{o}^{(3)}(c) ; \gamma\right)
\end{array}\right|
$$

Given an initial length of the cylinder, for each choice of $n$, there exists a non-trivial solution of the system (36)-(38) satisfying the prescribed boundary conditions for values of $\gamma$ at which the determinant vanishes. To find such values, we use a numerical root-finding scheme, such as the interval bisection method. For a growing tube, the critical growth value corresponding to the mode $n$, say $\gamma^{n}$ is the smallest value of $\gamma$ in the interval $(1, \infty)$ for which $\Delta(\gamma)=0$. The critical growth is then given by $\gamma^{c}=\min _{n}\left(\gamma^{n}\right)$.

\section{3-D Elasticity Results}

We first examine the effect of the outer layer thickness on the buckling behaviour. The curves in Figure 2 (a) show $\gamma^{n}$ as a function of the outer radius, $C$, for fixed inner radius, $B=1$, initial length, $L_{0}=100$, and shear modulus ratio (which we shall call the stiffness ratio), $\mu_{i} / \mu_{o}=100$. For each mode the corresponding growth curve is monotonic and approaches a constant value as $C$ increases (this is observable for $n>5$ in Figure 2(a), but the limits are not shown for lower modes). This is due to the fact that increasing the thickness of the outer layer results in a greater degree of transverse reinforcement acting on the inner layer, and the displacement of the surrounding medium is confined to a small region surrounding the inner layer. Within this region, the magnitude of the displacement decreases as $r$ increases so that material points far from the buckled filament are less displaced than those close to the material boundary. Thus, increasing the thickness of the outer layer beyond a certain amount has no effect on the resulting value of $\gamma^{n}$.

For each $C$ the critical growth $\gamma^{c}$ is given by $\gamma^{c}=\min _{n}\left(\gamma^{n}\right)$, as shown in Figure 2(b). Visual depictions of the critical mode in each region are shown in Figure 2(e). For the given parameter values, we see that close to $C=15$ a mode transition occurs, where the observed buckling mode suddenly switches from $n=1$ to $n=13$. A similar jump in buckling mode has been observed previously by Moulton and Goriely [15] in the case of a single layer tube subject to radial growth. We can consider the total energy of the system to be a combination of the bending energy of the inner cylinder and the elastic 'foundation' energy of the outer layer. For a one-layer tube $(C=B)$, we recover the classical Euler beam problem, and expect the mode $n=1$ to be selected. When $C$ is close to $B$, the foundation energy is small compared to the bending energy, therefore longer wavelength modes are selected, when compared to the case in which $C$ is large. As $C$ increases, the foundation energy of the outer layer plays a more significant role and the inner layer 'prefers' to undergo a higher degree of bending. Therefore, shorter wavelength modes are selected. Thus, we can interpret the transition point as the maximum thickness of the outer tube for which larger foundation displacements are preferable to a higher degree of bending.

Next, we consider the case of a fixed geometry with varying stiffness ratio, that is, we set $\mu_{o}=1$ and vary $\mu_{i}$. In Figure 2(c) we see that for each buckling mode, the critical growth value decreases monotonically as the stiffness ratio increases. This reflects the fact that a relatively soft outer layer is easier to displace than a relatively stiff outer layer. Thus, as $\mu_{i} / \mu_{o}$ increases, the amount of lateral reinforcement decreases so that less axial growth can occur before the onset of instability. We also observe that each curve approaches a constant value that is greater than or equal to 1 as $\mu_{i} / \mu_{o} \rightarrow \infty$. We can consider this limit to be identical to the scenario of the outer layer being absent altogether, which is precisely the Euler buckling problem [4]. Furthermore, the observed buckling mode is highly sensitive to variations in the stiffness ratio, particularly when $\mu_{i} / \mu_{o}<40$. In this region, shorter wavelength buckling modes are selected as the observed buckling mode because the associated bending energy cost 


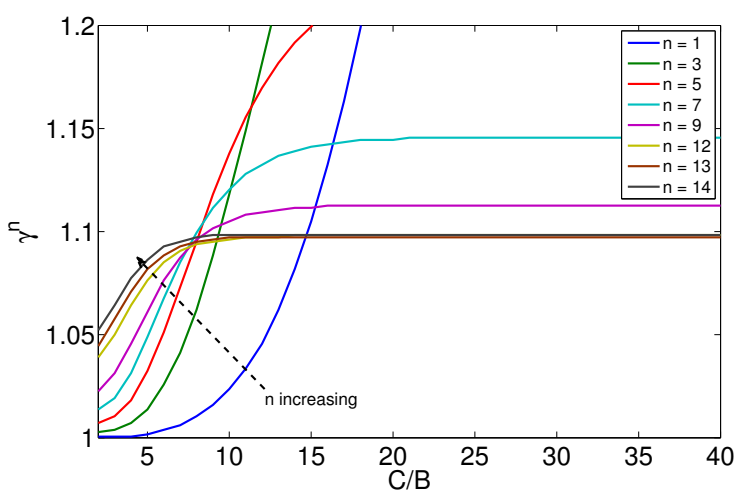

(a) $\mu_{i} / \mu_{o}=100, B=1, L_{0}=100$.

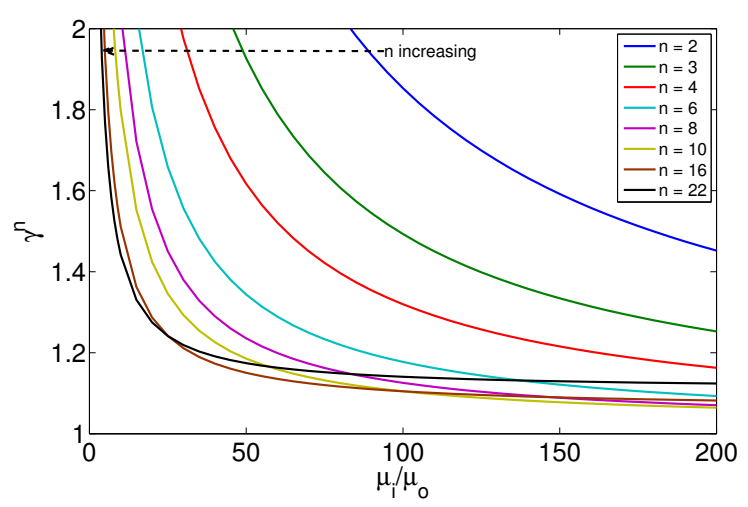

(c) $B=1, C=40, L_{0}=100$.

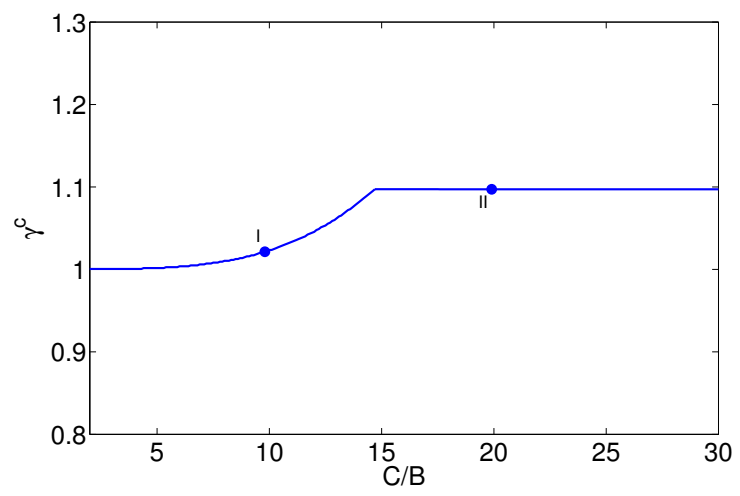

(b) $\mu_{i} / \mu_{o}=100, B=1, L_{0}=100$.

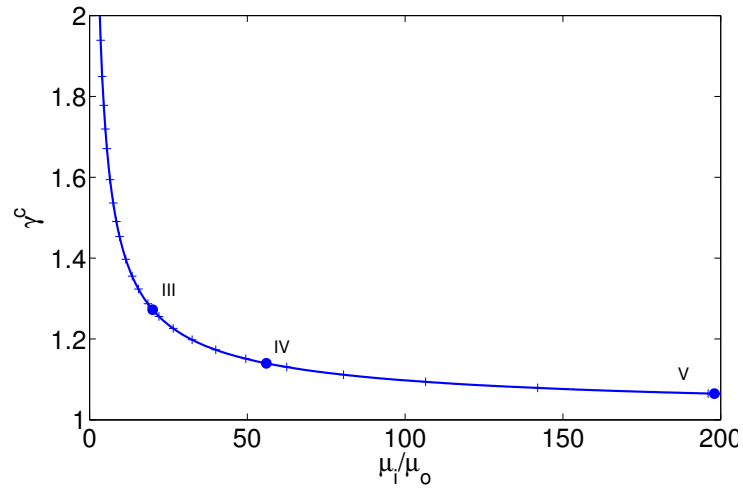

(d) $B=1, C=40, L_{0}=100$.

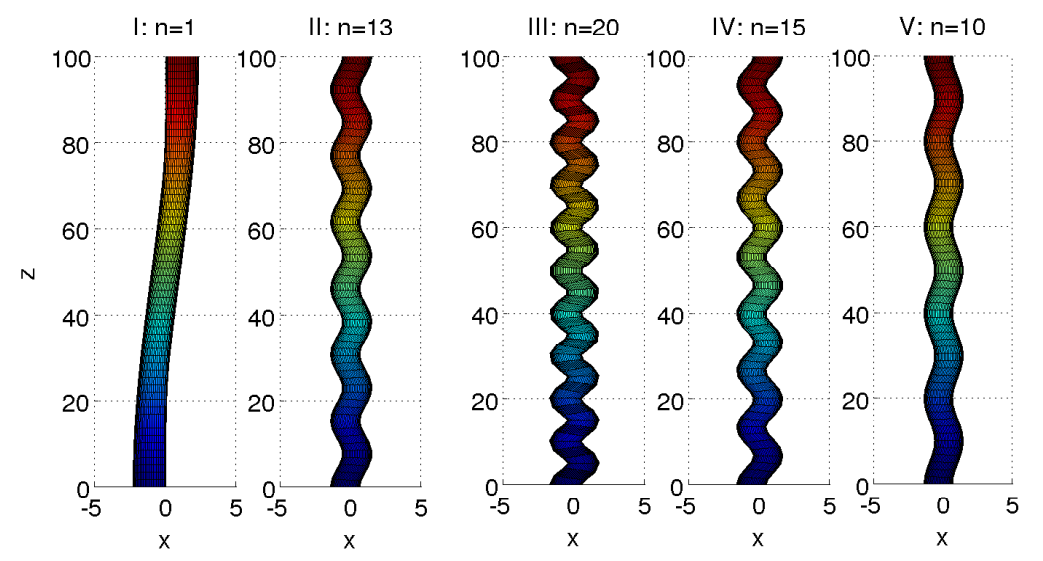

(e)

Figure 2: (a) Critical growth vs. relative thickness of layers for a range of modes between $n=1$ and $n=14$ and fixed stiffness ratio. (b) Overall critical growth vs. relative thickness of each layer. Around $k=15$ a mode transition occurs, where the observed buckled mode swtiches from one with a long wavelength to one with a short wavelength. (c) Critical growth vs. stiffness ratio $\mu_{i} / \mu_{o}$ for a range of modes between $n=1$ and $n=22$. (d) Overall critical growth vs. stiffness ratio. Crosses indicate transition points. We note the curve appears much smoother than that shown in (b) and there exist many more transition points. (e) Depictions of the buckled inner filament corresponding to each of the points marked in (b) and (d). The amplitude of the oscillations is determined by $\epsilon$ and has no physical significance. It has been chosen to illustrate the form of the structure. 


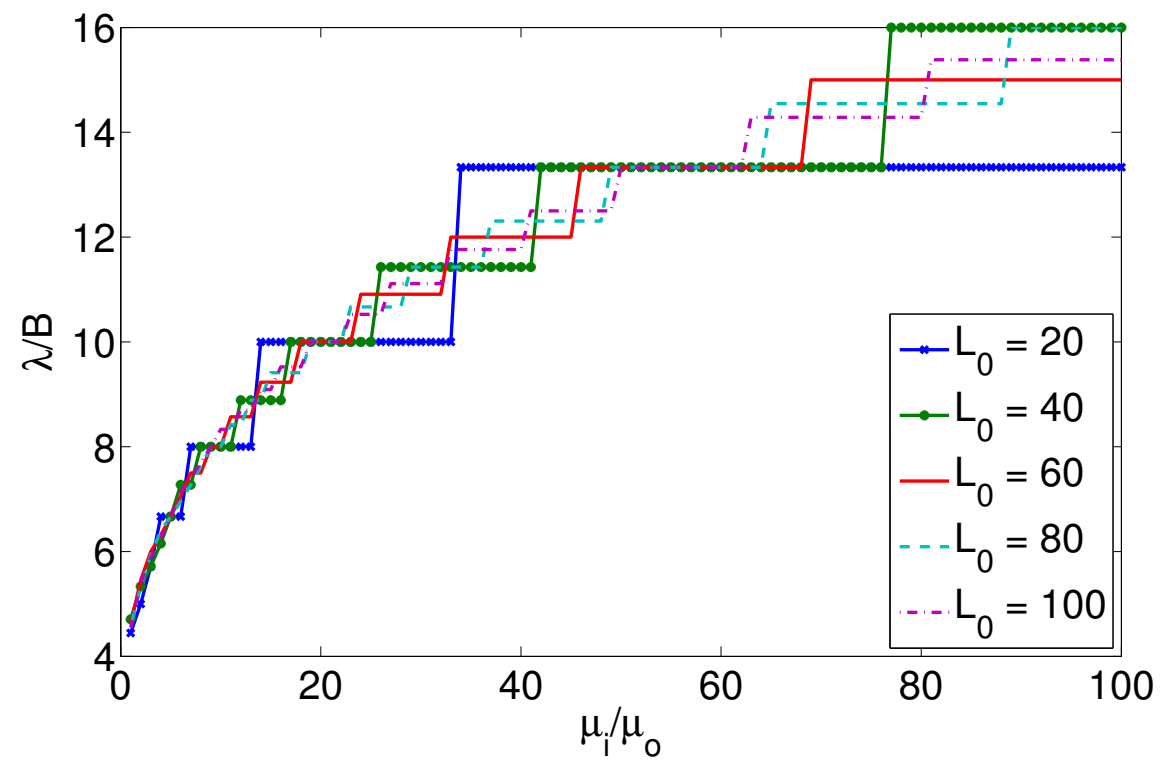

Figure 3: Observed buckling wavelength vs. stiffness ratio $\mu_{i} / \mu_{o}$ for $B=1, C=40$ and a range of initial lengths.

is low, whilst longer wavelength modes are selected as the relative stiffness and thus foundation energy of the outer layer decreases. The critical growth $\gamma^{c}$ as a function of $\mu_{i} / \mu_{o}$ is given in Figure $2(\mathrm{~d})$. We observe much 'smoother' transitions between the observed modes as $\mu_{i} / \mu_{o}$ varies, when compared to the case of varying $C$. Depictions of the inner cylinder are shown in Figure 2(e) for the indicated points in Figure 2(d).

Repeating our numerical procedure for different values of $L_{0}$ while maintaining $B$ and $C$ at fixed values, we find that the graphs of $\gamma^{c}$ against $\mu_{i} / \mu_{o}$ (not shown), are identical. This indicates that in the case of a filament embedded in a sufficiently thick outer layer, the critical growth value is independent of the initial length of the filament. Figure 3 shows the corresponding observed dimensionless buckling wavelength, $\lambda$, against the stiffness ratio for each value of $L_{0}$. We observe that the initial length does have some influence on the buckling wavelength, particularly when $B / L_{0}=O(1)$. However, this dependence arises due to our demand that $n$ is an integer, so that the wavelength must belong to a discrete set of values. As $L_{0}$ increases we observe smaller differences between the curves, so for large $L_{0}$ we may estimate the wavelength by fitting a smooth curve to the discretized results.

In Figure 4, the log-log plot of the dimensionless wavelength of the observed buckling mode against the stiffness ratio $\mu_{i} / \mu_{o}$ is shown for the case $L_{0}=100$. We observe that the slope is close to $1 / 4$, which indicates that the observed buckling wavelength and stiffness ratio are related by a power law, i.e. $\lambda \propto\left(\mu_{i} / \mu_{o}\right)^{1 / 4}$. This is consistent with a basic dimensional analysis an can already be found in the original work of Winkler [23,5]. We also consider the case $m=0$ (i.e. barrelling modes) for the parameter values $L_{0}=10, B=1, C=40$ and varying $\mu_{i} / \mu_{o}$. In the region of interest $\left(25<\mu_{i} / \mu_{o}<200\right)$, the critical growth $\min _{n}\left(\gamma^{n}\right)$ is greater than that corresponding to the critical growth in the case $m=1$. This justifies the assumption that slender filaments will always exhibit axial buckling rather than barrelling instabilities.

\subsection{WKB Approximation}

We are particularly interested in the case of a slender filament embedded in an infinite matrix, so that we can compare the results of the 3-D approach with those obtained using a rod-theory approach. Therefore, we seek analytical solutions of the equilibrium equations (36)-(38) in the case $C \rightarrow \infty$ by considering the problem of a neo-Hookean cylinder embedded in an infinite neo-Hookean matrix.

By substituting the relevant expressions for $\mathcal{L}_{i j k l}$ for a neo-Hookean material into (36)-(38) we obtain 


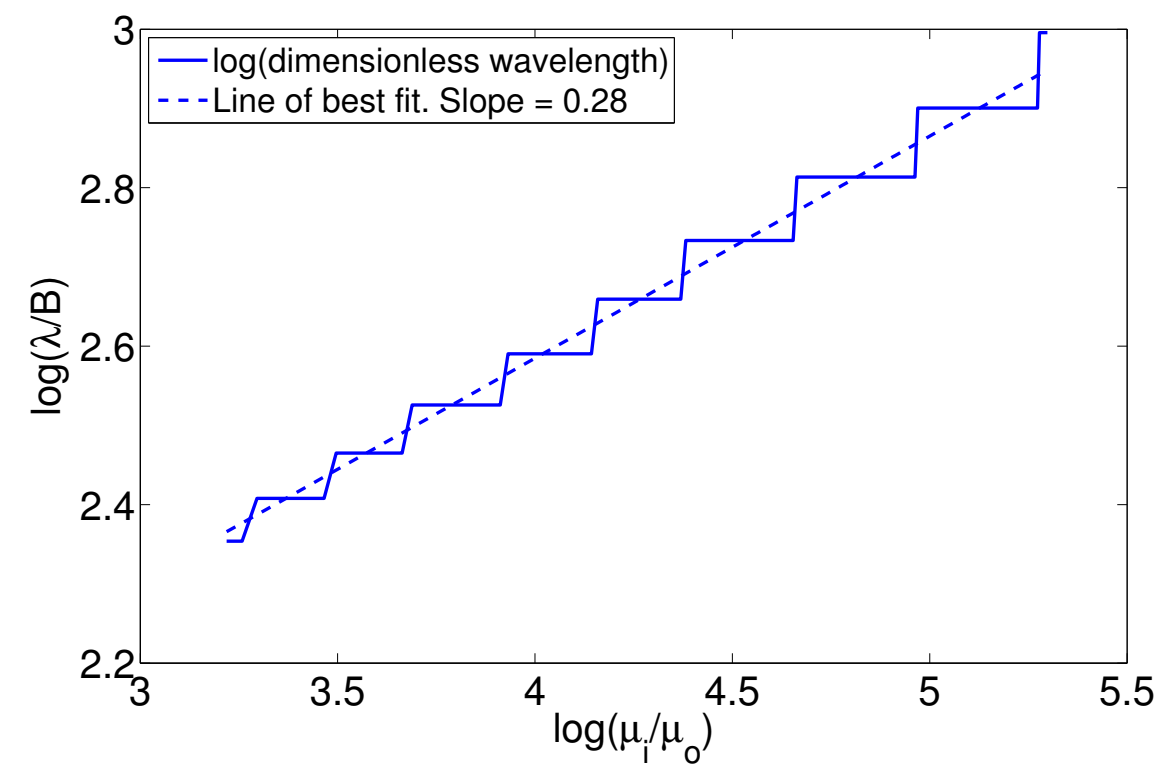

Figure 4: log-log plot of observed buckling wavelength vs. stiffness ratio $\mu_{i} / \mu_{o}$.

the following system of equations in the inner layer:

$$
\begin{aligned}
& 0=f^{\prime \prime}+\frac{1}{r} f^{\prime}-\left(\frac{n^{2}}{\gamma^{3}}+\frac{2}{r^{2}}\right) f-\frac{2}{r^{2}} g-\frac{1}{\gamma \mu_{i}} k^{\prime} \\
& 0=g^{\prime \prime}+\frac{1}{r} g^{\prime}-\left(\frac{n^{2}}{\gamma^{3}}+\frac{2}{r^{2}}\right) g-\frac{2}{r^{2}} f-\frac{1}{\gamma \mu_{i}} \frac{k}{r} \\
& 0=-f^{\prime \prime \prime}-\frac{2}{r} f^{\prime \prime}+\left(\frac{n^{2}}{\gamma^{3}}+\frac{2}{r^{2}}\right) f^{\prime}+\frac{n^{2}}{\gamma^{3} r} f-\frac{1}{r} g^{\prime \prime}+\frac{1}{r^{2}} g^{\prime}+\frac{n^{2}}{\gamma^{3} r} g+\frac{n^{2}}{\gamma \mu_{i}} k .
\end{aligned}
$$

In the outer layer, we define $\hat{\kappa}=1-\left(1-\gamma^{-1}\right) b^{2} r^{-2}$. The resulting system is then given by

$$
\begin{aligned}
0 & =\left(1-\left(1-\gamma^{-1}\right) \frac{b^{2}}{r^{2}}\right) f^{\prime \prime}+\left(2-\frac{1}{\hat{\kappa}}+2\left(1-\gamma^{-1}\right) \frac{b^{2}}{r^{2}}\right) \frac{f^{\prime}}{r}-\left(n^{2} r^{2}+\frac{2}{\hat{\kappa}}\right) \frac{f}{r^{2}}-\frac{2}{r^{2} \hat{\kappa}} g-\frac{1}{\mu_{o}} k^{\prime}, \\
0 & =-\left(1+\frac{1}{\hat{\kappa}}+\left(1-\gamma^{-1}\right) \frac{b^{2}}{r^{2}}\right) \frac{1}{r^{2}} f+\left(1-\left(1-\gamma^{-1}\right) \frac{b^{2}}{r^{2}}\right) g^{\prime \prime} \\
& +\left(1+\left(1-\gamma^{-1}\right) \frac{b^{2}}{r^{2}}\right) \frac{g^{\prime}}{r}-\left(n^{2} r^{2}+1+\frac{1}{\hat{\kappa}}+\left(1-\gamma^{-1}\right) \frac{b^{2}}{r^{2}}\right) \frac{g}{r^{2}}+\frac{1}{r \mu_{o}} k, \\
0 & =-\left(1-\left(1-\gamma^{-1}\right) \frac{b^{2}}{r^{2}}\right) f^{\prime \prime \prime}-\frac{2}{r} f^{\prime \prime}+\left(n^{2} r^{2}+1+\frac{1}{\hat{\kappa}}-3\left(1-\gamma^{-1}\right) \frac{b^{2}}{r^{2}}\right) \frac{f^{\prime}}{r^{2}} \\
& +\left(\frac{1}{\hat{\kappa}}-1+3\left(1-\gamma^{-1}\right) \frac{b^{2}}{r^{2}}+\frac{n^{2} r^{2}}{\hat{\kappa}}-b^{2} n^{2}\left(1-\gamma^{-1}\right)\right) \frac{f}{r^{3}}-\left(1-\left(1-\gamma^{-1}\right) \frac{b^{2}}{r^{2}}\right) g^{\prime \prime} \\
& +\left(1-3\left(1-\gamma^{-1}\right) \frac{b^{2}}{r^{2}}\right) \frac{1}{r^{2}} g^{\prime}+\left(n^{2} r^{2}-1+\frac{1}{\hat{\kappa}}+3\left(1-\gamma^{-1}\right) \frac{b^{2}}{r^{2}}\right) \frac{1}{r^{3}} g+\frac{n^{2}}{\mu_{o}} k .
\end{aligned}
$$


As in the case of a finite outer radius, we require finite radial stress as $r \rightarrow 0$ and

$$
\begin{aligned}
f_{i} & =f_{o}, \\
g_{i} & =g_{o}, \\
f_{i}^{\prime} & =f_{o}^{\prime}, \\
2 \gamma \mu_{i}\left(f_{i}^{\prime}-\frac{k_{i}}{\mu_{i}}\right) & =2 \mu_{o}\left(\hat{\kappa} f_{o}^{\prime}-\frac{k_{o}}{\mu_{o}}\right), \\
\gamma \mu_{i}\left(-\frac{f_{i}}{r}+g_{i}^{\prime}-\frac{g_{i}}{r}\right) & =\mu_{o} \hat{\kappa}\left(-\frac{f_{o}}{r}+g_{o}^{\prime}-\frac{g_{o}}{r}\right), \\
\gamma \mu_{i}\left(-f_{i}^{\prime \prime}-\frac{f_{i}^{\prime}}{r}+\left(1-\alpha^{2} r^{2}\right) \frac{f_{i}}{r^{2}}+\frac{g_{i}^{\prime}}{r}+\frac{g_{i}}{r^{2}}\right) & =\mu_{o} \hat{\kappa}\left(-f_{o}^{\prime \prime}-\frac{f_{o}^{\prime}}{r}+\left(1-\alpha^{2} r^{2}\right) \frac{f_{o}}{r^{2}}+\frac{g_{o}^{\prime}}{r}+\frac{g_{o}}{r^{2}}\right),
\end{aligned}
$$

at $r=b$. We replace the zero-stress condition at $r=c$ with the condition that $f_{o}, g_{o}, k_{o} \rightarrow 0$ as $r \rightarrow \infty$.

\subsubsection{Inner Layer}

Equations (57)-(59) can be solved exactly by using a method described in [2]. We proceed by introducting the potential functions $\Omega(r, \theta, z)$ and $\Psi(r, \theta, z)$ such that the displacements $u, v$ and $w$ are given by:

$$
u=\Omega_{r, z}+\frac{\Psi_{\theta}}{r}, \quad v=\frac{\Omega_{\theta, z}}{r}-\Psi_{r}, \quad w=-\nabla^{2} \Omega .
$$

Furthermore, we set

$$
\begin{aligned}
& \Omega=\omega(r) \cos \theta \sin \alpha z, \\
& \Psi=\psi(r) \sin \theta \cos \alpha z,
\end{aligned}
$$

so that substitution into (69) gives:

$$
f_{i}(r)=\alpha \omega^{\prime}+\frac{\psi}{r}, \quad g_{i}(r)=-\frac{\alpha}{r} \omega-\psi^{\prime}
$$

Then, eliminating $k$ from (57)-(59) we are able to solve the remaining equations exactly. Imposing the finite stress condition results in the following expressions for $\omega$ and $\psi$ :

$$
\omega=a_{1} I_{1}(\alpha r)+a_{2} I_{1}\left(\left(\frac{1}{\gamma}\right)^{\frac{3}{2}} \alpha r\right), \quad \psi=a_{3} I_{1}\left(\left(\frac{1}{\gamma}\right)^{\frac{3}{2}} \alpha r\right),
$$

where $I_{1}(r)$ is a modified Bessel function of the first kind. Therefore we have the following expressions for $f_{i}, g_{i}$ and $k_{i}$ :

$$
\begin{aligned}
f_{i} & =\alpha^{2} a_{1} I_{2}(\alpha r)+\frac{I_{1}}{r}\left(\alpha r\left(\frac{1}{\gamma_{3}}\right)^{\frac{3}{2}}\right)\left(a_{3}+a_{2} \alpha\right)+a_{2} \alpha^{2} I_{2}\left(\alpha r\left(\frac{1}{\gamma}\right)^{\frac{3}{2}}\right)\left(\frac{1}{\gamma_{3}}\right)^{\frac{3}{2}} \\
& +\frac{a_{1} \alpha}{r} I_{1}(\alpha r), \\
g_{i} & =-\frac{I_{1}}{r}\left(\alpha r\left(\frac{1}{\gamma}\right)^{\frac{3}{2}}\right)\left(a_{3}+a_{2} \alpha\right)-a_{3} \alpha I_{2}\left(\alpha r\left(\frac{1}{\gamma}\right)^{\frac{3}{2}}\right)\left(\frac{1}{\gamma}\right)^{\frac{3}{2}}-\frac{a_{1} \alpha}{r} I_{1}(\alpha r), \\
k_{i} & =-a_{1} \frac{\mu_{i}}{\gamma^{2}} \alpha^{3} I_{1}(\alpha r)\left(\gamma^{3}-1\right),
\end{aligned}
$$

where $a_{j}, j=1,2,3$, are constants. 


\subsubsection{Outer Layer}

Equations (60)-(62) do not yield an exact analytical solution. We consider the case of a high axial wavenumber, that is $\alpha \gg 1$ so that $\delta=\alpha^{-1} \ll 1$ and obtain an approximate solution using the WKB method. Note that we cannot guarantee a priori that for given $L_{0}$ the value of $\alpha$ corresponding to the observed buckling mode will indeed be large and this will be discussed later. Further details can be found in [6]. In short, we seek solutions of the form

$$
\left\{\begin{array}{l}
f_{o} \\
g_{o} \\
k_{o}
\end{array}\right\}=\left\{\begin{array}{c}
F \\
G \\
\alpha K
\end{array}\right\} \exp \left(\alpha \int^{r} s(x) d x\right) .
$$

Substituting this expression into the equilibrium system leads to the following forms of $f_{o}, g_{o}$ and $k_{o}$ at leading order:

$$
\begin{aligned}
f_{o} & =\frac{a_{4} \sqrt{r} \mathrm{e}^{-\alpha r}+a_{5} \mathrm{e}^{-\alpha \sqrt{r^{2}-\gamma+1}}\left(r^{2}-\gamma+1\right)^{1 / 4}}{\sqrt{\gamma-1}} \\
g_{o} & =\frac{a_{6} \mathrm{e}^{-\alpha \sqrt{r^{2}-\gamma+1}}}{\left(r^{2}-\gamma+1\right)^{1 / 4}}, \\
k_{o} & =\frac{a_{5} \alpha \mathrm{e}^{-\alpha r} \sqrt{\gamma-1}}{r^{3 / 2}}
\end{aligned}
$$

where $a_{j}, j=4,5,6$, are constants.

Substitution of (74)-(76) and (78)-(80) into the matching conditions (63)-(68) results in the matrix equation

$$
\text { M.a }=0,
$$

where $\mathbf{a}=\left(a_{1}, a_{2}, a_{3}, a_{4}, a_{5}, a_{6}\right)^{T}$ and $M_{i j}$ is the coefficient of $a_{j}$ in each boundary condition. For a given aspect ratio $B / L_{0}$ and stiffness ratio $\mu_{i} / \mu_{o}$ we select a buckling mode of interest (i.e. choose values for $m$ and $n$ ) and solve the following to find $\gamma^{n}$ :

$$
\operatorname{det}(\mathbf{M})\left(\gamma^{\mathrm{n}}\right)=0
$$

Again, the critical growth is then given by $\gamma^{c}=\min _{n}\left(\gamma^{n}\right)$.

\subsection{Comparison of Numerical and WKB Results}

We assess the accuracy of the WKB approximation by comparing the estimated critical growth values and corresponding wavelengths obtained using this method with the numerical results. As before, we assume $m=1$. In Figure 5 plots of $\gamma^{c}$ as a function of $\mu_{i} / \mu_{o}$ are shown for five different values of $L_{0}$, together with the corresponding plot obtained numerically. We see that the estimates lie close to the numerical curves, which indicates that our approximation provides a good estimate of the critical growth.

In Figure 6 the estimated observed buckling wavelength is plotted along with the corresponding numerical value. We see good agreement with the numerical results, even though the largest value of $\alpha$ is approximately given by $0.4 \pi$. Our approximation was based on the assumption that the wavenumber, $\alpha$, is large, but we conclude that the approximation gives good estimates of $\gamma^{c}$, even when $\alpha=O(1)$. The WKB method also recovers the power law relationship between wavelength and stiffness ratio obtained from the numerical results and the work of [3].

\section{Elastic Rod Approach}

The numerical results in Section 3 indicate that in the buckled configuration, spatial oscillations within the body are confined to a single plane. Therefore, an alternative approach is to use a rod-theory formulation, which treats the inner layer as an elastic rod embedded in an infinite medium subject to axial growth and transverse displacements. We use the general formalism of morphoelastic rods [16] for this particular problem. The full rod analysis also shows that the first instability is planar. Therefore, we restrict our attention to planar deformations of a growing rod on an elastic foundation and compare both approaches. 


\subsection{Setup}

We consider a stress-free straight unshearable rod of length $L_{0}$ lying in the $(x, y)$ plane. The rod's centreline lies along the $x$-axis, and it is confined between two rigid plates a fixed distance, $L_{0}$, apart. The rod is subjected to axial growth, $\gamma$, so that in the absence of the plates, its unstressed (natural) length is $L=\gamma L_{0}$. However the axial constriction supplied by the plates results in the rod becoming compressed, until it buckles. Let $S_{0}$ and $s$ denote the arc length of the rod in the initial and current configurations respectively. Additionally, let $S$ denote arc length in the stress-free grown configuration i.e. the configuration the grown rod would adopt if the plates were removed and define $\beta=d s / d S$. We use $\kappa$ and $\theta$ to denote the curvature of the rod and angle between the rod's centreline and the $x$-axis respectively.

Assuming the system is in static equilibrium and neglecting body couples, the principles of conservation of linear and angular momentum leads to

$$
\begin{gathered}
\frac{\partial \mathbf{n}}{\partial S}+\mathbf{f}=0, \\
\frac{\partial \mathbf{m}}{\partial S}+\frac{\partial \mathbf{r}}{\partial S} \times \mathbf{n}=0,
\end{gathered}
$$

where $\mathbf{m}=\left(0,0, m_{z}\right)$ is the resultant moment, $\mathbf{n}=\left(n_{x}, n_{y}, 0\right)$ is the resultant force, $\mathbf{f}$ are the body forces per unit unstressed natural length. We assume further that the outer matrix acts as a Winkler foundation, that is we use a linear relationship between the transverse displacement and transverse body force acting on the rod due to the surrounding matrix, so that the transverse component of traction acting on the rod is given by $-k_{f} y$, where $k_{f}$ is the foundation modulus parameter, which characterises the resistive force of the surrounding medium. Finally, we close the problem by imposing the standard constitutive relations for the rod

$$
\begin{gathered}
m_{z}=E I \beta \kappa=E I \beta \frac{d \theta}{d s}, \\
n_{x} \cos \theta+n_{y} \sin \theta=E A(\beta-1),
\end{gathered}
$$

where $E, I$ and $A$ are defined in Table 1 . Note that since $(\beta-1)$ measures the stretch in the rod, the last equation is simply a form of Hooke's law when uniaxial deformations are considered. Substitution into the equilibrium equations then gives

$$
\left\{\begin{array}{l}
x^{\prime}=\gamma \beta \cos \theta \\
y^{\prime}=\gamma \beta \sin \theta \\
n_{x}^{\prime}=0 \\
n_{y}^{\prime}=\gamma \beta k_{f} y \\
E I \theta^{\prime \prime}+\gamma\left(x^{\prime} n_{y}-y^{\prime} n_{x}\right)=0
\end{array}\right.
$$

where primes denote differentiation with respect to $S_{0}$. The boundary conditions are given by:

$$
x(0)=0, \quad x\left(L_{0}\right)=L_{0}, \quad \theta(0)=\theta\left(L_{0}\right)=0, \quad n_{y}(0)=n_{y}\left(L_{0}\right)=0 .
$$

\subsection{Buckling}

We proceed by considering small perturbations around the straight but compressed straight rod solution. That is, we introduce perturbations of size $0<\epsilon \ll 1$ and make the following expansions:

$$
\begin{aligned}
& x=x_{0}+\epsilon x_{1}+\ldots, \\
& y=y_{0}+\epsilon y_{1}+\ldots, \\
& \theta=\epsilon \theta_{1}+\ldots, \\
& n_{x}=n_{x 0}+\epsilon n_{x 1}+\ldots, \\
& n_{y}=n_{y 0}+\epsilon n_{y 1}+\ldots, \\
& \beta=\beta_{0}+\epsilon \beta_{1}+\ldots .
\end{aligned}
$$


At leading order, we obtain the system

$$
\left\{\begin{array}{l}
x_{0}^{\prime}=\gamma \beta_{0} \\
y_{0}^{\prime}=0 \\
n_{y 0}^{\prime}=\gamma \beta_{0} k_{f} y_{0} \\
E I \theta_{0}^{\prime \prime}+\gamma\left(x_{0}^{\prime} n_{y 0}-y_{0}^{\prime} n_{x}\right)=0
\end{array}\right.
$$

and the constitutive relation yields

$$
n_{x 0}=E A\left(\beta_{0}-1\right) .
$$

We can see that there exists a non-trivial solution, given by

$$
x_{0}=S, \quad \beta_{0}=\frac{1}{\gamma}, \quad y_{0}=0, \quad n_{y 0}=0 .
$$

This solution corresponds with the finite, pre-buckled solution derived in the 3-D elasticity problem in Section 2.3.1.

To examine the stability of the straight solution we consider the $\mathrm{O}(\epsilon)$ system

$$
\left\{\begin{array}{l}
x_{1}^{\prime}=\gamma \beta_{1} \\
y_{1}^{\prime}=\gamma \beta_{0} \theta_{1} \\
n_{y 1}^{\prime}=\gamma \beta_{0} k_{f} y_{1} \\
E I \theta_{1}^{\prime \prime}+\gamma\left(n_{y 1}-y_{1}^{\prime} n_{x 0}\right)=0
\end{array}\right.
$$

and

$$
\beta_{1}=0,
$$

which follows from the constitutive relation. Substitution leads to the following fourth-order ordinary differential equation for $\theta_{1}$

$$
E I \theta_{1}^{\prime \prime \prime \prime}+\gamma\left(k_{f} \theta_{1}-n_{x 0} \theta_{1}^{\prime \prime}\right)=0 .
$$

We seek solutions of the form $\theta_{1} \sim \mathrm{e}^{n \pi i S_{0} / L_{0}}$ (real part assumed), where $n$ is a positive integer. Substitution into (94) results in the following quadratic equation for $n^{2}$ :

$$
\frac{E I \pi^{4}}{L_{0}^{4}} n^{4}+\frac{\gamma n_{x 0} \pi^{2}}{L_{0}^{2}} n^{2}+\gamma k_{f}=0
$$

Note that in the absence of the outer layer $k_{f}=0$ and we recover the classic Euler beam theory (see e.g. Chapter 4.9 .3 of [12]). Substitution of (90) into (95) then yields the following expression for $\gamma$ :

$$
\gamma(n)=\frac{E I n^{4} \pi^{4}+L_{0}^{2} E A n^{2} \pi^{2}}{L_{0}^{2} E A n^{2} \pi^{2}-L_{0}^{4} k_{f}}=\frac{E I \alpha^{4}+E A \alpha^{2}}{E A \alpha^{2}-k_{f}}
$$

We wish to find the buckling mode that minimises the critical growth. Therefore we solve $d \gamma / d n=0$ to find some $n^{*}$, which will lie between two consecutive integers, say $n_{1}$ and $n_{2}$. The critical growth is then given by $\gamma^{c}=\min \left(\gamma\left(n_{1}\right), \gamma\left(n_{2}\right)\right)$ and the corresponding $n_{j}, j=1,2$, is the observed buckling mode. Note that in the limit $L_{0} \rightarrow \infty$, the difference between successive modes, $((n+1) \pi-n \pi) / L_{0}=\pi / L_{0}$ tends to zero. In this case the critical growth value is given precisely by the value of $\gamma$ for which (95) has real roots. Therefore we have

$$
E^{2} A^{2}(\gamma-1)^{2}=4 E I \gamma k_{f},
$$

which yields

$$
\gamma^{c}=1+\frac{2 I k_{f}}{E A^{2}}\left(1+\sqrt{1+\frac{E A^{2}}{I k_{f}}}\right), \quad \lambda=2 \pi\left(\frac{E I}{\gamma k_{f}}\right)^{1 / 4}
$$

\section{Connecting the approaches}

As discussed in the Introduction, there is no rational method to obtain the parameter $k_{f}$, despite the fact that it is widely used in many rod-theory problems. We relate this parameter to measurable properties of the system by comparing the results of the rod-theory approach described in the previous section with the results of the 3 -D formulation. To do so, we first compare the roles of the various parameters 


\begin{tabular}{|c|c||c|c|}
\hline 3-D Model & Role of Parameter(s) & rod-theory Model & Role of Parameter(s) \\
\hline$L_{0}$ & Initial length of cylinder & $L_{0}$ & Initial length of rod \\
$l$ & Deformed length of cylinder (pre-bifurcation) & $l$ & Compressed length of rod \\
$\gamma$ & Axial growth & $A=\pi B^{2}, I=\pi B^{4} / 4$ & Cross-sectional area and moment of inertia \\
$B$ & Cross-sectional radius of stress-free inner cylinder & $E=3 \mu_{i}$ & Young's modulus of rod \\
$\mu_{i}$ & Shear modulus of inner layer & $x, y, \theta$ & Geometry of buckled rod \\
$u, w$ & Post-bifurcation displacements & $k_{f}$ & Foundation modulus \\
$\mu_{0}$ & Shear modulus of outer layer & $S_{0}$ & Arc length of undeformed rod \\
$z$ & Axial co-ordinate & &
\end{tabular}

Table 1: Relationships between the parameters used in each modelling approach.

involved in each modelling approach. A summary of the correspondence between the parameters of the two models is shown in Table 1. In particular, we note that the parameters $\mu_{o}$ and $k_{f}$ are related to each other, though the exact relationship between them is unknown. We investigate whether a simple, linear relationship provides agreement with the 3 -D elasticity approach. That is, we postulate

$$
k_{f}=C_{1} \mu_{o},
$$

where $C_{1}$ is a constant. In the case of a neo-Hookean strain energy density, such an ansatz is a natural choice for the proposed relationship. For a rod with a circular cross-section we have $A=\pi B^{2}$ and $I=\pi B^{4} / 4$. For each choice of $C_{1}$ we solve (95) to find the observed buckling mode of the rod and its corresponding critical growth value, $\gamma^{c}$, in terms of $\mu_{i} / \mu_{o}$. We then estimate $k_{f}$ by seeking the value of $C_{1}$ that minimises the sum of squares between these data and the corresponding results obtained numerically in Section 3.

Figures 5 and 6 show the critical growth and wavelength plots obtained using the WKB approximation and rod-theory approach, together with the corresponding numerical results obtained in Section 3 for ease of comparison. The fitting of the results from the rod-theory approach with the corresponding results from the 3-D approach was repeated for different values of $L_{0}$ to determine whether $C_{1}$ is, in fact, dependent upon the geometry of the rod (though only the plots corresponding to the case $L_{0}=100$ are shown, because other values of $L_{0}$ give rise to almost identical results). The region of fitting used was $25<\mu_{i} / \mu_{o}<200$. We restrict attention to this region because stiffness ratios lower than $\mu_{i} / \mu_{o}=200$ are most common throughout nature, and when $\mu_{i} / \mu_{o}<25$ the axial strain is greater than $20 \%$. In the case of low $L_{0}(\approx 10)$, there is some variation of $C_{1}$ with $L_{0}$. However, this is precisely the regime in which we expect the rod-theory assumptions to break down, since $B / L_{0}=O(1)$. As $L_{0}$ increases, $C_{1}$ becomes geometry-independent and is approximately equal to $2.8 \pi$. Figure 5 shows good agreement in the critical growth curves in the region $25<\mu_{i} / \mu_{o}<200$ for this choice of constant, with the maximum relative difference between the two curves being approximately 0.02 , when $\mu_{i} / \mu_{o}=25$. When the observed buckling wavelength is considered, as shown in Figure 6, the rod-theory approach tends to slightly underestimate the observed wavelength, compared to the numerical results.

There are two possibles causes for the the slight difference between the two results. First, we note that there exists a potentially significant difference between the two modelling approaches. In the 3-D elasticity formulation, we adopted the assumption of incompressibility. Therefore, as the inner cylinder grows subject to the axial constraint, its cross-sectional area must increase to preserve the overall volume of the grown structure. In the elastic rod model, we can account for this cross-sectional change in the case of bending energy by modifying $A$ and $I$ accordingly. However, a key assumption of our approach is that the transverse force $n_{y}$ acts on the rod's centreline. Therefore, as long as the rod remains straight it experiences the axially compressive force only. This simplification thus affects the energy required by the rod to displace the surrounding matrix. Second, in Figure 2(c) we note that the critical growth values for the various axial buckling modes are very close. This suggests that small differences in the critical growth value could result in significant qualitative differences in the state of the rod post-bifurcation, as is the case with our two modelling approaches. Nevertheless, the agreement between the two approaches is generally good, and it is interesting that a single value of $C_{1}$ is sufficient to characterise the relationship between the shear modulus of the elastic matrix and the transverse pressure it applies to the growing filament inside. We also compare our estimate of $k_{f}$ with those given in (1) and (2). The parameter $l$ is 


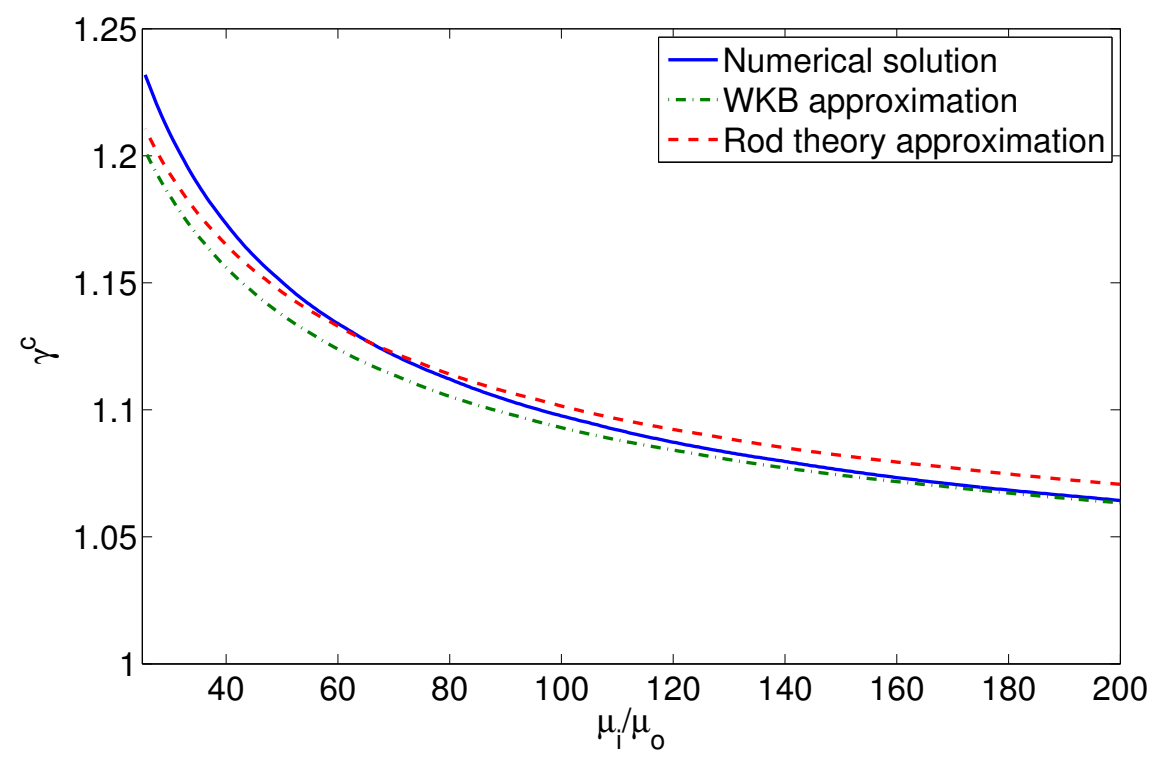

Figure 5: Comparison of critical growth value vs. stiffness ratio curves in a fixed geometry for the two modelling approaches. The parameter values used are $E=3 \mu_{i}=3, L_{0}=100, B=1$, and $C=40$ (the latter applies to 3-D elasticity approach only). The value of $C_{1}$ that minimises the sum of squares between the rod-theory and numerical results is $2.8 \pi$.

taken to be 10 (since Figure 3 shows the observed dimensionless buckling wavelength to be of the order 10). This yields a values $\alpha_{e 1}=5.2 \pi$ which is about twice too large. If we set $C_{1}=\alpha_{e 1}$ and substitute into (96), this leads to significant underestimation of the critical growth value, when compared with the numerical results of the 3-D approach. The estimate $\alpha_{e 2}$ is also unreliable because it is dependent upon the length of the filament and as the length of the filament increases, $k_{f} \rightarrow 0$ leading to the unphysical behaviour that an infinite filament in an elastic matrix would be equivalent to a beam without matrix.

So far, our estimates of $k_{f}$ have been obtained by fitting the critical growth curves in a relevant parameter regime. However by combining the results of the rod-theory approach and asymptotic approximation obtained in Section 3.1 we are able to derive a relationship between the shear modulus of the elastic matrix and the resulting transverse force acting on the embedded rod. By equating the relevant solution of (82) with the right hand side of (98(a)), an estimate of $C_{1}$ in terms of $\mu_{i} / \mu_{o}$ is given by

$$
C_{1}=3 \pi \frac{\left(1-\gamma^{c}\right)^{2}}{\gamma^{c}} \frac{\mu_{i}}{\mu_{o}}
$$

where $\gamma^{c}$ satisfies (82). As an example, we consider the case $\mu_{i}=200$. The corresponding critical growth obtained using the WKB method is given by $\gamma^{c} \approx 1.06$ and substitution into (100) yields the estimate $C_{1} \approx 2.3 \pi$, which is in good agreement with our fitted value of $C_{1}$.

\section{Discussion}

Within the framework of 3-D elasticity, we have presented a model to predict the critical axial growth of a two-layer cylinder constrained between two rigid plates. This has allowed us to study the buckling of slender filaments embedded in a thick elastic matrix. By taking the limit of an infinitely thick outer layer we obtained an exact solution to the inner layer equations and an analytic approximation to the outer layer equations via the WKB method. We have demonstrated that this is comparable with the finite-thickness case, since for all $n, \gamma^{n}$ approaches a constant value as $C \rightarrow \infty$ for fixed $B$. We have also compared this model with an approach making use of elastic rod-theory, under the assumptions that the rod lies within an infinite Winkler foundation and that the shear modulus of the foundation, $\mu_{o}$, is 


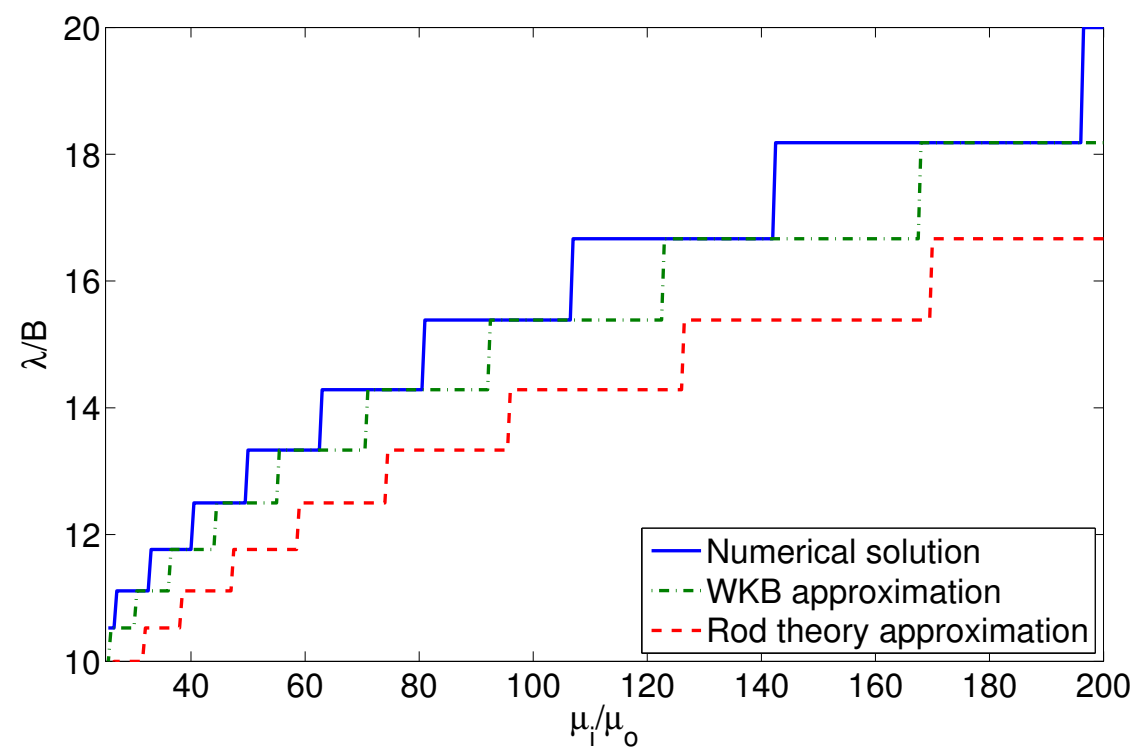

Figure 6: Comparison of observed buckling wavelength vs. stiffness ratio in a fixed geometry for the two modelling approaches. The parameter values used are identical to those used in Figure 5 and $C_{1}=2.8 \pi$.

directly proportional to the transverse pressure, $k_{f}$.

An advantage of using the 3-D elasticity formulation is that it can easily be adapted for a wide range of strain energy densities. We now briefly consider the case of the outer layer comprising a Mooney-Rivlin material, to determine whether the value of $C_{1}$ is affected by a second material parameter. The strain energy density in the outer layer is now given by:

$$
W_{o}=\frac{C_{10}}{2}\left(\alpha_{1}^{2}+\alpha_{2}^{2}+\alpha_{3}^{2}\right)+\frac{C_{01}}{2}\left(\alpha_{1}^{2} \alpha_{2}^{2}+\alpha_{2}^{2} \alpha_{3}^{2}+\alpha_{3}^{2} \alpha_{1}^{2}\right)
$$

The shear modulus is then defined to be $\mu_{o}=C_{10}+C_{01}$. In the case $C_{01}=0$ we recover the neo-Hookean model. To investigate the role of each parameter in this model, the numerical scheme described in Section 3 was repeated for the case $B=1, C=40, L_{0}=100$ and various choices of $C_{10}$ and $C_{01}$ such that $\mu_{o}=1$. The resulting critical growth and wavelength curves were almost identical to the curves obtained in the neo-Hookean case, though they exhibited slight variation for lower values of $\mu_{i} / \mu_{o}\left(i . e . \mu_{i} / \mu_{o}<40\right)$. We therefore conclude that for a fixed geometry, the estimate for $k_{f}$ is well captured by the tangent shear modulus around the unstressed state and that no significant changes are observed in third-order elasticity (which for an incompressible material is equivalent to Mooney-Rivlin). Therefore, we do not expect the foundation modulus to depend on the nonlinear properties of the strain-energy density function close to the bifurcation.

A drawback of the 3-D approach is that it requires numerical methods to solve the resulting equilibrium equations, even in the case of a simple strain energy density. Moreover, the method of determinants can become unreliable for large or small values of $\gamma$ due to the exponential growth of one or more of the $\mathbf{y}^{(i)}$ in (52). One way of overcoming this limitation is to use an alternative numerical scheme, such as the compound matrix method [6], though this is still computationally intensive. We are also able to make use of the WKB-approximation, which allows investigation of a wider range of parameter space. This is particularly useful should we wish to investigate deformations that give rise to a combination of axial and circumferential buckling modes, such as those observed in solid tumour capillaries [13].

The elastic rod-theory approach is comparatively quick to implement and is widely used throughout existing literature. Moreover, this approach allows the deformation of the filament to be tracked for different loads and beyond the point at which buckling occurs. The main drawback of the rod-theory approach is that the constant $k_{f}$ is difficult to determine, whilst the 3 -D approach only requires knowledge of the elastic properties of the two layers.

Both modelling approaches recover the power law relationship between the stiffness ratio and buck- 
ling wavelength, first derived by Winkler. They also show that as the relative stiffness of the outer layer increases, the critical axial growth decreases monotonically and the wavelength of the buckled configuration increases. Moreover, their resulting critical growth values are close to one another in the region of interest, and the critical growth is independent of the initial length of the filament. We have also shown that the two approaches can be used to provide an estimate of the foundation modulus parameter, $k_{f}$. The WKB method allows us to estimate $\gamma^{c}$ in terms of $\mu_{i}$ and $\mu_{o}$, and Equation (100) can then be used to provide an estimate of $C_{1}$ that shows good agreement with the corresponding value obtained by fitting the results of the two approaches. Therefore we suggest the following estimate for the foundation modulus parameter of a Winkler elastic foundation of shear modulus $\mu_{o}$ to be

$$
k_{f}=2.8 \pi \mu_{o},
$$

independent of the length or radius of the filament.

\section{Acknowledgements}

This publication is based on work supported in part by Award No. KUK-C1-013-04, made by King Abdullah University of Science and Technology (KAUST) to the Oxford Centre for Collaborative Applied Mathematics (OCCAM). AG is a Wolfson/Royal Society Merit Award Holder and acknowledges support from a Reintegration Grant under EC Framework VII. SO'K is supported by the Univeristy of Oxford Systems Biology Doctoral Training Centre, which is funded by EPSRC. We would like to thank Luis Dorfmann and Yibin Fu for interesting discussions on instabilities in non-linear elasticity. SO'K, DM, and $\mathrm{AG}$ are members of OCCAM, DM is a member of the Centre for Mathematical Biology (CMB) and SW and AG are members of the Oxford Centre for Industrial Applied Mathematics (OCIAM). The support of these centres is gratefully acknowledged.

\section{References}

[1] M. Ben Amar and A. Goriely. Growth and instability in elastic tissues. J. Mech. Phys. Solids, 53:2284-2319, 2005.

[2] D. Bigoni and M. Gei. Bifurcations of a coated, elastic cylinder. International Journal of Solids and Structures, 38:5117-5148, 2001.

[3] C. P. Brangwynne, F. C. MacKintosh, S. Kumar, N. A. Geisse, J. Talbot, L. Mahadevan, K. K. Parker, D. E. Ingber, and D. A. Weitz. Microtubules can bear enhanced compressive loads in living cells because of lateral reinforcement. J. Cell Biol., 173:733-741, 2006.

[4] L. Euler. Sur la force des colonnes. Vol. 13 Berlin, Germany: Memoires de l'Academie de Berlin., 1759 .

[5] L. Frýba. History of Winkler foundation. Vehicle System Dynamics, 24:sup1:7-12, 1995.

[6] Y. B. Fu and M. Sanjarani Pour. WKB method with repeated roots and its application to the buckling analysis of an everted cylindrical tube. SIAM Journal of Applied Mathematics, 62(6):1856$1871,2002$.

[7] Y. C. Fung. What are the residual stresses doing in our blood vessels? Annals of Biomedical Engineering, 19:237-249, 1991.

[8] A. Goriely, M. Destrade, and M. Ben Amar. Instabilities in elastomers and in soft tissues. Q. J. Mech. Appl. Math, 59:615-630, 2006.

[9] A. Goriely, D. E. Moulton, and R. Vandiver. Elastic cavitation, tube hollowing, and differential growth in plants and biological tissues. EuroPhys. Lett., 91:18001, 2010.

[10] A. Goriely and R. Vandiver. On the mechanical stability of growing arteries. IMA Journal of Applied Mathematics, 75:549-570, 2010. 
[11] A. Goriely, R. Vandiver, and M. Destrade. Nonlinear Euler buckling. Proc. R. Soc. A, 464:3003-3019, 2008.

[12] P. Howell, G. Kozyreff, and J. Ockendon. Applied Solid Mechanics. Cambridge University Press, 1st edition, 2009.

[13] J. MacLaurin, S. J. Chapman, G. W. Jones, and T. Roose. The buckling of capillaries in solid tumours. Proc. R. Soc. A, 468:4123-4145, 2012.

[14] D. E. Moulton and A. Goriely. Possible role of differential growth in airway wall remodeling in asthma. J. Appl. Physiol., 110:1003-1012, 2010.

[15] D. E. Moulton and A. Goriely. Circumferential buckling instability of a growing cylindrical tube. J. Mech. Phys. Solids, 59:525-537, 2011.

[16] D. E. Moulton, T. Lessinnes, and A. Goriely. Morphoelastic Rods Part I: A single growing elastic rod. J. Mech. Phys. Solids, 61:398-427, 2013.

[17] T. Murmu and S. C. Pradhan. Buckling analysis of a single-walled carbon nanotube embedded in an elastic medium based on nonlocal elasticity and Timoshenko beam theory and using DQM. Physica E, pages 1232-1239, 2009.

[18] R. W. Ogden. Non-Linear Elastic Deformations. Dover, 1st edition, 1997.

[19] E. K. Rodriguez, A. Hoger, and A. D. McCulloch. Stress-dependent finite growth in soft elastic tissues. J. Biomechanics, 27:455-467, 1994.

[20] W. L. Shan, Z. Chen, C. P. Broedersz, A. A. Gumaste, W. O. Soboyejo, and C. P. Brangwynne. Attenuated short wavelength buckling and force propagation in a biopolymer-reinforced rod. Soft Matter, 9:194-199, 2012.

[21] R. Vandiver and A. Goriely. Tissue tension and axial growth of cylindrical structures in plants and elastic tissues. EuroPhys. Lett., 84:58004, 2008.

[22] R. Vandiver and A. Goriely. Differential growth and residual stress in cylindrical elastic structures. Phil. Trans. R. Soc. A, 367:3607-3630, 2009.

[23] E. O. Winkler. Die Lehre von der Elasticitaet und Feistigkeit. Theil 1, 2. H. Dominicus, Prag, 1867.

[24] E. O. Winkler. Vorträge über Eisenbahnbau. Heft 1, 2. H. Dominicus, Prag, 1867. 



\section{RECENT REPORTS}

13/02 Drop spreading and penetration into pre-wetted powders

Marston

Sprittles

Zhu

$\mathrm{Li}$

Vakarelski

Thoroddsen

13/03 On the mechanics of thin films and growing surfaces

Holland

Kosmata

Goriely

Kuhl

13/04 Spatially Partitioned Embedded Runge-Kutta Methods

Ketcheson

Macdonald

Ruuth

13/05 Simple computation of reaction-diffusion processes on point clouds

Macdonald

Merriman

Ruuth

13/06 A Volume-Based Method for Denoising on Curved Surfaces

Biddle von Glehn

Macdonald

März

13/07 Porous squeeze-film flow

Knox

Wilson

Duffy

McKee

13/08 Diffusion of finite-size particles in confined geometries

Bruna

Chapman

13/09 Mathematical analysis of a model for the growth of the bovine corpus luteum

Prokopiou

Byrne

Jeffrey

Robinson

Mann

Owen

13/10 Capillary deformations of bendable films

Schroll

Adda-Bedia

Cerda

Huang

Menon

Russell

Toga

Vella

Davidovitch

13/11 Twist and stretch of helices: All you need is Love

Đuričković

Goriely

Maddocks

13/12 Switch on, switch off: stiction in nanoelectromechanical switches

Wagner

Vella

13/13 Pinning de-pinning and re-pinning of a slowly varying rivulet 
13/19 On a poroviscoelastic model for cell crawling

Kimpton

Whiteley

Waters

Oliver

13/20 Complexity Plots

Thiyagalingam

Walton

Duffy

Trefethen

Chen

13/21 Glyph-based video visualization for semen analysis

Duffy

Thiyagalingam

Walton

Smith

Trefethen

Kirkman-Brown

Gaffney

Chen

13/22 RBF multiscale collocation for second order elliptic boundary

Farrell value problems

Wendland

13/23 Na/K pump regulation of cardiac repolarization: Insights from a systems biology approach

Bueno-Orovio

Sánchez

Pueyo

Rodriguez

13/24 Cellular blebs - pressure-driven, axisymmetric, membrane protrusions

Woolley

Gaffney

Oliver

Baker

Waters

Goriely

Copies of these, and any other OCCAM reports can be obtained from:

Oxford Centre for Collaborative Applied Mathematics

Mathematical Institute

24 - 29 St Giles'

Oxford

OX1 3LB

England

www.maths.ox.ac.uk/occam 